\title{
Coupled Interannual Variability of the Hadley and Ferrel Cells
}

\author{
PABLO ZURITA-GOTOR \\ Universidad Complutense de Madrid, and Instituto de Geociencia UCM-CSIC, Madrid, Spain \\ PABLo ÁlVAREZ-ZAPATERo \\ Universidad Complutense de Madrid, Madrid, Spain
}

(Manuscript received 2 November 2017, in final form 12 March 2018)

\begin{abstract}
This work investigates the covariability in the strength of the Hadley and Ferrel cells on interannual time scales using reanalysis data. A significant correlation is found in both hemispheres only during boreal winter. For other seasons, only the outermost (subtropical) part of the Hadley cell is correlated with changes in the extratropical eddy momentum fluxes, as the eddies are unable to penetrate into the deep tropics. During boreal winter, the northern Hadley cell variability is driven by extratropical planetary momentum fluxes, but the mean meridional circulation response is primarily found below the level of maximum climatological outflow. Instead, at upper levels, changes in the zonal wind dominate the response to the anomalous eddy forcing. During austral winter, the southern Hadley cell is shielded from the extratropical eddy fluxes and its variability displays some of the characteristics of the angular momentum-conserving solution.
\end{abstract}

\section{Introduction}

The mean meridional circulation (MMC) is a key element of the atmospheric general circulation, with the Hadley and Ferrel cells delineating the different dynamical regimes at work in the tropics and extratropics. Yet in spite of the different dynamics of these two cells, the global momentum balance requires some degree of covariability between them. As eddy fluxes in the subtropical upper troposphere transport momentum between both regions, the MMC provides the main dynamical mechanism for bringing this momentum forcing down to the surface, where it can be equilibrated by friction. (In the tropics the MMC meridional momentum transport may also be important, so the eddy momentum forcing need not be balanced locally.)

Motivated by this picture, the present work aims to assess the covariability between Hadley and Ferrel cell strength on interannual time scales. To the best of our knowledge, this question has not been investigated before in the literature, though previous studies have addressed the related question of the impact of eddy driving on Hadley cell strength variability (Caballero

\footnotetext{
Corresponding author: Pablo Zurita-Gotor, pzurita@alum. mit.edu
}

2007, hereafter C07). As is well known, the mean Ferrel cell is forced by transient and planetary eddy stresses associated with the meridional propagation of Rossby waves in the extratropical upper troposphere (Chang 1996). Although the Hadley cell has been traditionally regarded as the response to localized convection over the ITCZ, meridionally propagating Rossby waves also force a thermally direct circulation in the regions where they are dissipated. Walker and Schneider (2006) and Schneider and Bordoni (2008) have argued using idealized model results that this mechanism may be important for driving the Hadley cell during some seasons. The two paradigms may be compatible and relevant over different regions if the inner, equatorward part of the Hadley cell is driven by tropical heating, and its outer, subtropical part is forced by eddy stresses.

On the other hand, the mechanisms driving the climatological Hadley cell and its interannual variability are not necessarily the same. For instance, it would be possible for the variability of a thermally forced Hadley cell to be driven by the eddy momentum fluxes if these fluxes were much more variable than the tropical heating. Using reanalysis data, $\mathrm{C} 07$ studied the impact of eddy forcing on the Hadley cell during boreal winter, finding significant driving of the northern (southern) Hadley cell variability by planetary (synoptic) eddies. Several 
subsequent studies on the impact of the extratropical circulation on the Hadley cell have focused on its extent rather than on its strength, finding significant Southern Hemisphere correlations during austral summer (Kang and Polvani 2011; Ceppi and Hartmann 2013).

To the best of our knowledge the impact of eddy forcing on Hadley cell strength in other seasons than boreal winter remains unstudied, an omission suggesting that eddies are only important during that season. This will be confirmed by our analysis, which shows that the maximum Hadley overturning is significantly correlated with Ferrel cell strength only during December through February (DJF). We analyze why this is the case and investigate whether eddy momentum fluxes can still play a role in the variability of the outer Hadley cell region. This paper is organized as follows. Section 2 describes the data used and the methods of analysis. Section 3 investigates the covariability between the Ferrel cell and different Hadley indices, and section 4 uses the anomalous tropical momentum balance to interpret these results. Section 5 concludes with a short discussion.

\section{Data and methods}

We use in this study zonal and meridional wind data and pressure velocity at $2.5^{\circ}$ horizontal resolution from the ERA-Interim reanalysis dataset (Dee et al. 2011) from 1979 to 2015 (37 yr), and monthly sea surface temperature (SST) data from the Extended Reconstructed SST dataset, version 5 (Huang et al. 2015). For each year, seasonal means are calculated averaging the data for each of the standard seasons: DJF, March through May (MAM), June through August (JJA), and September through November (SON). Eddy momentum fluxes are computed by averaging zonally and throughout the season the products of 6-hourly eddy anomalies, where eddies are defined as deviations from the zonal mean.

The main analysis variable is the mass streamfunction for the mean meridional circulation, which we calculate by integrating the meridional mass flux down from the top of the atmosphere:

$$
\psi=2 \pi a \cos \phi \int_{0}^{p} \bar{v} \frac{d p}{g},
$$

where $\bar{v}$ is the zonal-mean meridional wind, $\phi$ is latitude, and $a$ is the radius of Earth.

We study the interannual relations between the strength of the tropical and extratropical circulations using indices. First, we define the Hadley and Ferrel cell boundaries as the zeros of the vertically integrated mass streamfunction from the surface to $200 \mathrm{hPa}$, which captures the bulk of the tropospheric circulation. The use of the vertical integral is meant to eliminate sensitivity to the choice of an arbitrary reference level (Davis and Birner 2017), and the zeros are calculated using linear interpolation. We use as an index of Ferrel cell intensity the latitudinal maximum (in absolute value) of the vertically integrated mass streamfunction over the midlatitude domain defined by the zero crossings. This definition provides a measure of the net overturning independent of the meridional circulation shifts that dominate Ferrel cell variability.

For the Hadley cell, we use three different indices. All these indices are defined in terms of the net overturning at each latitude (vertical maximum of the mass streamfunction, in absolute value) but the results are robust to using other definitions like the vertically integrated streamfunction or its midtropospheric value. The first index, Hadmax, is defined as the maximum overturning within the Hadley cell domain as in $\mathrm{C} 07$ and similar to the Ferrel index above. Since this maximum overturning is located deep into the tropics and may not reflect changes in the strength of the outer parts of the Hadley cell, we define two other indices integrating the vertical streamfunction maximum meridionally over the whole Hadley cell (Hadint index) or poleward of the maximum overturning (Hadsub index). For these integrals, we use as integration limits the climatological Hadley cell boundaries (defined by the zero crossings of the mean vertically integrated streamfunction) rather than the actual boundaries for the given year to prevent contamination from circulation shifts. We found that when the actual time-dependent boundaries are used for integration, Hadsub variability is associated with Hadley cell expansion/contraction and more strongly correlated with annular mode variability (Ferrel cell shifts) than with Ferrel cell strengthening (results based on the Hadint index are similar with fixed and varying integration limits).

To aid the interpretation of the results, we also introduce an index uvmax measuring the tropicalextratropical momentum exchange. This index is defined as the latitudinal maximum of the uppertroposphere (400-150-hPa integral) poleward eddy momentum flux, motivated by the fact that this flux tends to peak at the Hadley cell boundary as discussed by Vallis et al. (2015). (The results are similar if we use the eddy momentum flux at a fixed latitude instead.)

To analyze the data we use simple Pearson correlations and linear regression analysis. It is well known that the Hadley cell has expanded in recent decades (e.g., Davis and Rosenlof 2012), though the magnitude and significance of the tropical circulation trends depend on the reanalysis product and the specific metric considered 
TABLE 1. Correlations between the three Hadley indices and the Ferrel index in the Southern Hemisphere, between the Hadley indices in both hemispheres, and between the three Hadley indices and the Ferrel index in the Northern Hemisphere for the seasons indicated and annually, based on detrended data with ENSO variability filtered out (correlations without ENSO filtering are shown in parentheses). Values significant at the $99 \%$ level are shown with bold face.

\begin{tabular}{|c|c|c|c|c|c|c|c|}
\hline & \multicolumn{3}{|c|}{$\mathrm{H}-\mathrm{F}(\mathrm{SH})$} & \multirow{2}{*}{$\frac{\mathrm{H}-\mathrm{H}}{\operatorname{Hadmax}}$} & \multicolumn{3}{|c|}{$\mathrm{H}-\mathrm{F}(\mathrm{NH})$} \\
\hline & Hadmax & Hadint & Hadsub & & Hadmax & Hadint & Hadsub \\
\hline DJF & $0.48(0.48)$ & $0.60(0.54)$ & $0.46(0.48)$ & $0.05(0.32)$ & $\mathbf{0 . 4 3}(0.22)$ & $0.54(0.51)$ & $0.70(0.71)$ \\
\hline MAM & $0.01(-0.01)$ & $0.14(0.16)$ & $0.48(0.48)$ & $0.48(0.52)$ & $0.11(0.09)$ & $0.18(0.12)$ & $0.21(0.17)$ \\
\hline JJA & $0.05(0.03)$ & $0.28(0.33)$ & $0.42(0.50)$ & $0.18(0.18)$ & $0.09(-0.05)$ & $0.12(0.01)$ & $0.24(0.37)$ \\
\hline SON & $0.16(-0.05)$ & $0.29(0.24)$ & $0.36(0.36)$ & $0.36(0.33)$ & $0.15(0.16)$ & $0.32(0.29)$ & $0.42(0.42)$ \\
\hline ANN & $0.33(0.27)$ & $0.23(0.21)$ & $0.47(0.48)$ & $0.35(\mathbf{0 . 4 6})$ & $-0.02(-0.07)$ & $0.18(0.14)$ & $0.36(0.36)$ \\
\hline
\end{tabular}

(Nguyen et al. 2013). Some studies have also found significant trends in Hadley cell strength (Stachnik and Schumacher 2011), which appear to be weaker for ERAInterim than for other reanalysis products. The analyses presented in this paper have been carried out using detrended time series but we note that our results are insensitive to inclusion of the trends (which do not exceed the $99 \%$ significance level). Because the ENSO impact on the tropical and extratropical overturning circulation has been described elsewhere (Seager et al. 2005), we focus in this study on non-ENSO variability. Except where otherwise indicated, we remove the ENSO signal from the data prior to the analysis by subtracting the linear ENSO response obtained by regression of the dynamical fields on the Niño-3.4 index, standardized over the period of study.

Since all indices are standardized prior to the regression analysis, the amplitude of the regression maps shown in this paper corresponds to one standard deviation of the index time series. We assess the statistical significance of these maps using two-tailed $t$ tests for the correlations, assuming that all 37 years are independent.

\section{Hadley-Ferrel covariability}

Table 1 shows interannual Pearson correlations between the detrended Ferrel cell index and the three Hadley indices introduced above when ENSO variability is removed (raw correlations including ENSO variability are also shown in parentheses for reference). When the maximum Hadley overturning (Hadmax index) is considered, significant correlations at the $99 \%$ level are found during boreal winter (DJF) in both hemispheres, consistent with the results of C07. However, no significant correlations are found during other seasons or in the annual mean. Although this paper focuses on Hadley-Ferrel covariability, we note that the Hadley cells in both hemispheres are also significantly correlated during MAM.

Correlations increase when the integrated Hadley index Hadint is used, but not enough to become significant.
In contrast, when the subtropical Hadley index Hadsub is used, the Hadley-Ferrel correlation becomes significant in the Southern Hemisphere during austral winter and in both hemispheres during fall. However, the $\mathrm{NH}$ summer correlation and the spring correlations in both hemispheres are still not significant. The correlations are similar whether ENSO variability is removed or not.

Figure 1 provides a more complete view of the coupled Hadley-Ferrel variability by showing the fraction of interannual streamfunction variance explained by the Hadley and Ferrel indices for some select seasons. In all cases, the positive sign of the correlations implies simultaneous intensification/weakening of both cells, and only values significant at the $95 \%$ level are shaded.

During austral summer, the SH Hadley and Ferrel cells have comparable intensity and each cell explains a significant fraction $(30 \%-35 \%)$ of the adjacent cell variability (Figs. 1a,b). The region with significant correlations has a deep structure and extends over the maxima in both cells. In contrast, during boreal summer, correlations between the northern Hadley and Ferrel cells are weak and not significant (not shown).

Figures $1 \mathrm{c}$ and $1 \mathrm{~d}$ show correlations with the northern and southern Ferrel indices during the boreal and austral winters, respectively. In both cases there is a significant subtropical Hadley cell signal, so that Ferrel cell strengthening is associated with an increase of the subsidence at the Hadley cell edge. However, during northern winter (DJF; Fig. 1c), the region of significant Ferrel cell correlation extends deeper into the tropics than during southern winter, consistent with the correlations in Table 1 . In both cases the region of influence is confined vertically below the level of maximum poleward flow, for reasons explained in the next section.

Similar to austral winter, only the subtropical part of the southern Hadley cell is correlated with Ferrel cell intensity during austral fall (MAM; Fig. 1e). The same is true during SON, but correlations are weaker in this case (see Fig. 3a). Similar results are obtained for the northern equinox seasons (not shown). Finally, Fig. 1f shows the correlation 

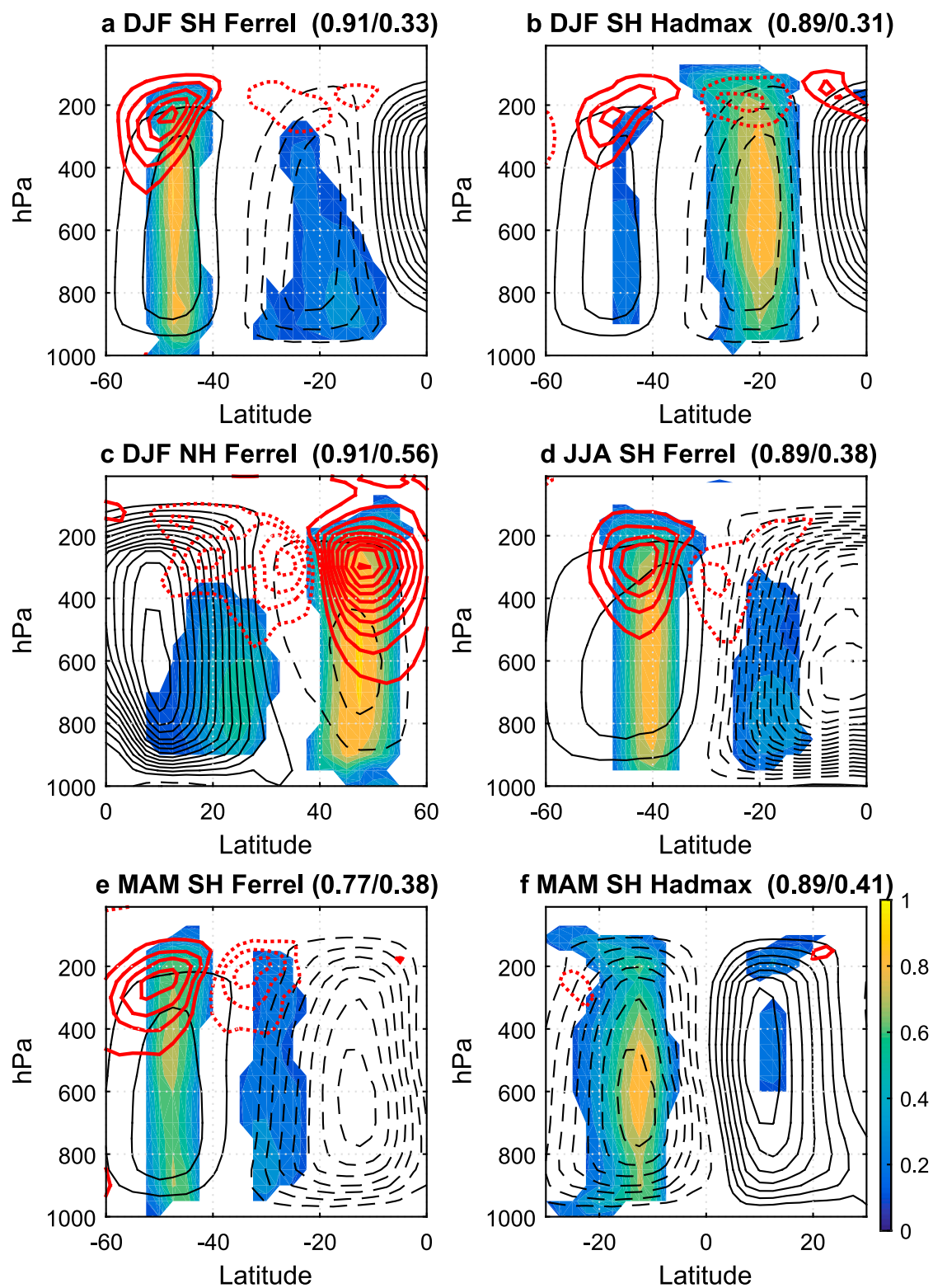

FIG. 1. For the seasons and indices indicated in panel titles: climatological-mean streamfunction (black contours, with negative contours dashed; contour interval: $1.5 \times 10^{10} \mathrm{~kg} \mathrm{~s}^{-1}$ ), fraction of streamfunction variance $r^{2}$ explained by the index (shading; only values significant at the $95 \%$ level are shown), and regressed poleward eddy momentum flux convergence (red contours, with negative contours dotted; contour interval: $0.1 \mathrm{~m} \mathrm{~s}^{-1} \mathrm{day}^{-1}$ ). The numbers in parentheses in all panel titles list the maximum explained variance over the driving cell and the adjacent cell.

between both Hadley cells during MAM. In contrast with the winter Hadley-Ferrel correlations, the covariability is now maximized over the upper troposphere.

\section{a. Eddy momentum forcing}

The regressed eddy meridional momentum convergence on the circulation indices (red contours in Fig. 1) is consistent with the driving of the coupled Hadley-Ferrel variability by the eddy momentum fluxes. On the other hand, the regressed eddy heat flux pattern is mostly limited to the extratropics and only significant in some seasons/hemispheres (not shown). This suggests that if eddy heat fluxes play any role in driving Hadley cell variability, it is only indirectly through their impact on eddy momentum fluxes rather than through their direct thermal forcing. 
Differences in the eddy momentum flux regression pattern also explain the stronger Hadley-Ferrel coupling during winter in the Northern than in the Southern Hemisphere, as Figs. 1c and 1d show that the regressed fluxes penetrate deeper in the tropics in the former. This is consistent with the arguments of Bordoni and Schneider (2008), as the upper-troposphere zonal winds are easterly in the deep tropics during boreal summer. Comparing Figs. 1c and 1d, we can see that the Hadley cell ascent is farther displaced into the summer hemisphere during JJA [see Shaw (2014) for a more complete description of this season] than during DJF, and the maximum Hadley overturning is also closer to the equator, effectively shielding the circulation from the influence of extratropical eddies.

Figure $2 \mathrm{a}$ shows the vertically integrated (surface to $150 \mathrm{hPa}$ ) momentum flux convergence, with Figs. $2 \mathrm{~b}$ and $2 \mathrm{c}$ showing its planetary/synoptic decomposition ( $k=1-3$ and $k \geq 4$, respectively; note that our definition of "synoptic" also includes medium-scale waves). It is apparent that the deeper eddy penetration during northern winter is due to the planetary eddies, as synoptic eddies actually penetrate farther in the $\mathrm{SH}$ than in the NH. The important role played by planetary eddies might also explain why Ferrel cell variability is only correlated with the maximum Hadley overturning during DJF in the $\mathrm{NH}$, as the planetary divergence of momentum in the deep tropics is significantly weaker in other seasons (e.g., Fig. 3b in Shaw 2014).

The deeper influence of planetary eddies is consistent with the slower phase speeds of these eddies, which allows them to propagate farther into the tropics (Randel and Held 1991). Figure 2d overlays the regressed eddy meridional divergence by synoptic/ planetary eddies (blue/maroon contours) on the climatological zonal wind during boreal winter. The bulk of the synoptic eddy absorption is found near the jet core, with the planetary divergence peaking at slower speeds on both sides of the jet (the equatorward maximum is stronger). A third planetary divergence maximum at even slower speeds is found at upper levels near the tropical tropopause. This location suggests that the divergence maximum might be associated with intertropical momentum exchange (Dima et al. 2005) rather than with tropical-extratropical interactions. To isolate this contribution, we decompose the planetary divergence into two components: one due to the equatorward momentum fluxes in the deep tropics, and another one associated with the poleward fluxes in the subtropics. The three contributions to the total divergence: synoptic (blue), extratropical planetary (maroon), and intertropical planetary (magenta) are shown in Fig. 2e. We will use the same color conventions for the diagnostics presented in the next section.

\section{b. Spring-fall asymmetry}

It is intriguing that autumn Ferrel correlations with the subtropical Hadley index are more significant than spring correlations in both hemispheres. A possible source for this asymmetry is the stratospheric circulation, which has very different character and interannual variability during fall and spring. It is possible that the interannual variability in the polar vortex may modulate some of the interannual Ferrel cell variability, masking the variability driven by tropical-extratropical momentum exchange.

To test this idea, we have defined an index (uvmax) to measure the tropical-extratropical momentum flux, computed as described in section 2. Not surprisingly, Table 2 shows that this index is very strongly correlated with the Ferrel cell index for all seasons. However, it is interesting that these correlations are lowest (though still highly significant) in the NH during JJA and in both hemispheres during spring, which suggests that the low Hadley-Ferrel correlations during these seasons may be partly due to the weakened link between Ferrel cell variability and subtropical eddy momentum flux. This is supported by the enhanced correlations between the uvmax index and the subtropical Hadley index in Table 2, as the correlations between the two are now significant at the $99 \%$ level in all seasons and both hemispheres.

The weak Hadley-Ferrel correlation in the NH during JJA likely reflects the fact that the extratropical circulation is too weak and poleward-displaced during this season, so that monsoon dynamics are more important than baroclinic eddies for driving Hadley cell variability and subtropical eddy momentum fluxes (Rodwell and Hoskins 2001). To investigate the source of the springfall asymmetry, Fig. 3 compares regressions based on the southern Ferrel cell index and the southern uvmax index during austral spring (SON). By construction, the latter index is associated with stronger and deeper eddy flux of easterly momentum flux into the tropics, consistent with the larger fraction of Hadley cell variability explained (cf. Figs. 3a and 3b). Examining other fields, there are also striking differences between the regressed zonalwind patterns on both indices. Figure 3 shows that Ferrel cell intensification is significantly associated with polar vortex strengthening (or late polar vortex breakdown), consistent with our speculation that stratospheric dynamics might be important for interannual Ferrel cell variability during spring. In contrast, regression on the uvmax index produces no significant polar wind anomalies, and Ferrel cell variability appears instead to be 

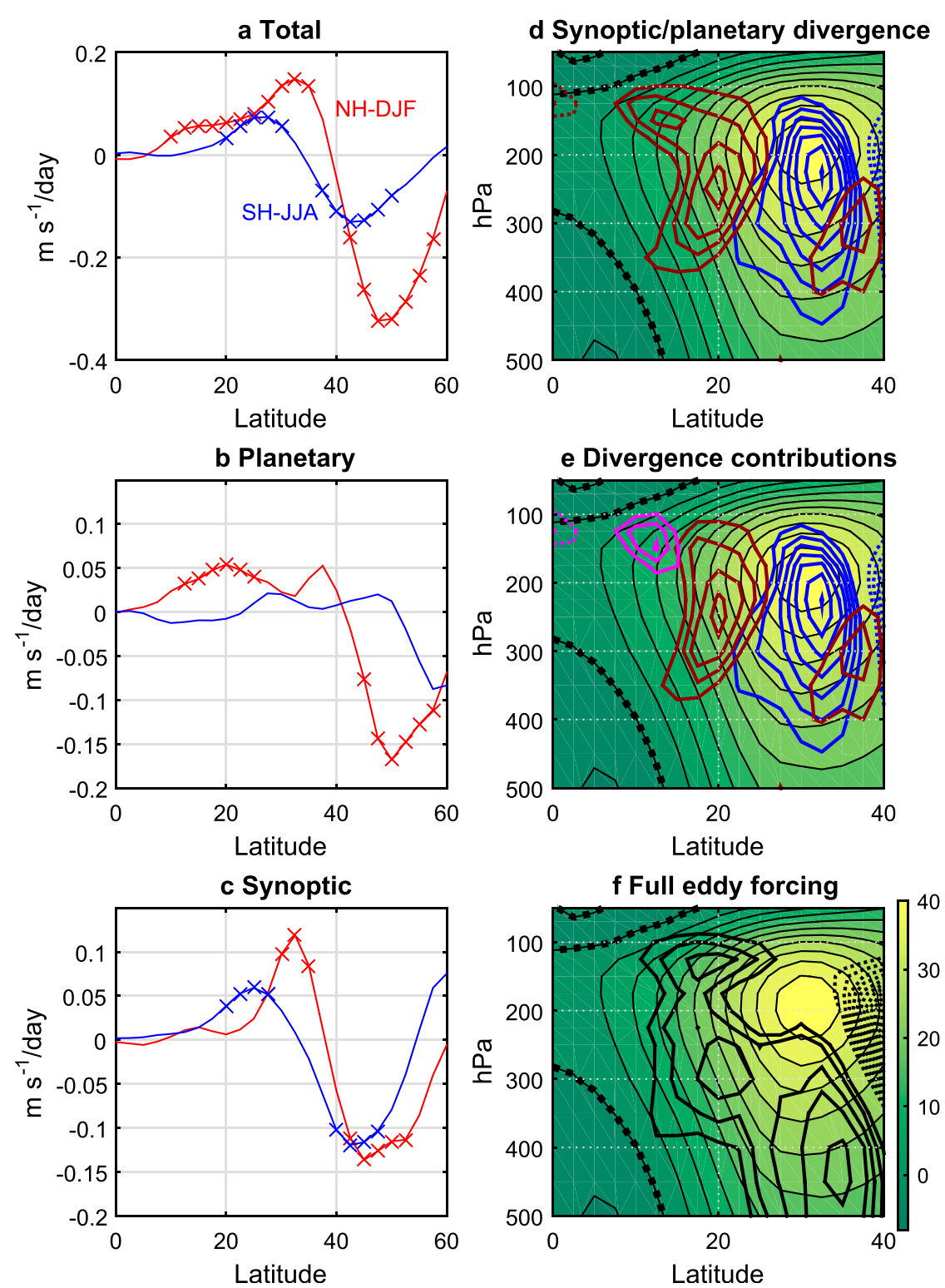

FIG. 2. (a) Vertically averaged (surface to $150 \mathrm{hPa}$ ) eddy momentum flux divergence in the $\mathrm{NH}$ (red) and SH (blue; latitudes in absolute value) during their respective winters, regressed on the corresponding Ferrel indices. Values significant at the $90 \%$ level are emphasized with crosses. (b) As in (a), but for planetary eddies $(k=1-3)$. (c) As in (a), but for synoptic eddies $(k \geq 4)$. (d) For the NH during boreal winter, climatological zonal wind (shading in $\mathrm{m} \mathrm{s}^{-1}$; the zero contour is shown with black thick-dotted line) and regressed meridional eddy momentum flux divergence on the Ferrel index. Blue (maroon) contours are used for synoptic (planetary) eddies, with negative contours dotted (contour interval:0.05 m s$~^{-1}$ day $^{-1}$ ). (e) As in (d), but splitting the planetary divergence into contributions by extratropical (maroon) and intertropical (magenta) eddies. (f) As in (d), but for the total (meridional and vertical) eddy momentum flux divergence for all eddies (thick black).

associated with tropical-extratropical momentum exchange (note the significant zonal-wind anomalies in the tropical troposphere). In the next section we investigate how the tropospheric tropical zonal wind responds to this momentum forcing, focusing on the winter seasons.

\section{Structure of the response and anomalous momentum balance}

It is striking in Figs. 1c, $1 \mathrm{~d}$, and $3 \mathrm{~b}$ that over the tropics, the bulk of the mean meridional circulation 
TABLE 2. Correlations between the maximum eddy momentum flux in the subtropics (uvmax) and the Ferrel cell index (Ferrel), the maximum Hadley index (Hadmax), and the subtropical Hadley index (Hadsub) for the Southern and Northern Hemispheres, based on detrended data with ENSO variability filtered out. Values significant at the $99 \%$ level are shown with bold face.

\begin{tabular}{lccccccc}
\hline & \multicolumn{2}{c}{ Southern Hemisphere } & & \multicolumn{2}{c}{ Northern Hemisphere } \\
\cline { 2 - 3 } \cline { 7 - 8 } \cline { 8 - 8 } & Ferrel & Hadmax & Hadsub & & Ferrel & Hadmax & Hadsub \\
\hline DJF & $\mathbf{0 . 8 2}$ & $\mathbf{0 . 4 0}$ & $\mathbf{0 . 5 5}$ & & $\mathbf{0 . 8 3}$ & $\mathbf{0 . 6 8}$ & $\mathbf{0 . 8 1}$ \\
MAM & $\mathbf{0 . 7 0}$ & 0.27 & $\mathbf{0 . 6 9}$ & & $\mathbf{0 . 6 6}$ & 0.28 & $\mathbf{0 . 4 2}$ \\
JJA & $\mathbf{0 . 7 6}$ & 0.11 & $\mathbf{0 . 5 7}$ & & $\mathbf{0 . 5 3}$ & 0.38 & $\mathbf{0 . 5 5}$ \\
SON & $\mathbf{0 . 6 6}$ & $\mathbf{0 . 4 8}$ & $\mathbf{0 . 6 7}$ & & $\mathbf{0 . 7 8}$ & 0.19 & $\mathbf{0 . 5 5}$ \\
ANN & $\mathbf{0 . 6 6}$ & $\mathbf{0 . 4 4}$ & $\mathbf{0 . 5 5}$ & & $\mathbf{0 . 7 3}$ & 0.22 & $\mathbf{0 . 5 6}$ \\
\hline
\end{tabular}

response to the eddy meridional momentum divergence is found at levels below the forcing. In this section we use the momentum balance to analyze the structure of the tropical circulation response in more detail.
In the extratropics, where the Rossby number is small, the meridional flow in the free troposphere is generally collocated with the eddy momentum divergence/ convergence in equilibrium. However, this is not necessarily the case in the tropics, where relative vorticity is comparable to planetary vorticity, and anomalous eddy divergence may also be balanced by changes in relative vorticity. In the free troposphere, we may approximate the momentum balance as

$$
(f+\bar{\xi}) \bar{v}-\bar{\omega} \bar{u}_{p}=S,
$$

where the overbar stands for the zonal mean, $f+\bar{\xi}$ is absolute vorticity, $\bar{u}_{p}$ is vertical shear, and $S$ is the net (meridional plus vertical) eddy momentum divergence. The second term on the left-hand side (MMC vertical advection) is usually neglected in this balance (e.g., Schneider 2006), but this is the only term that can
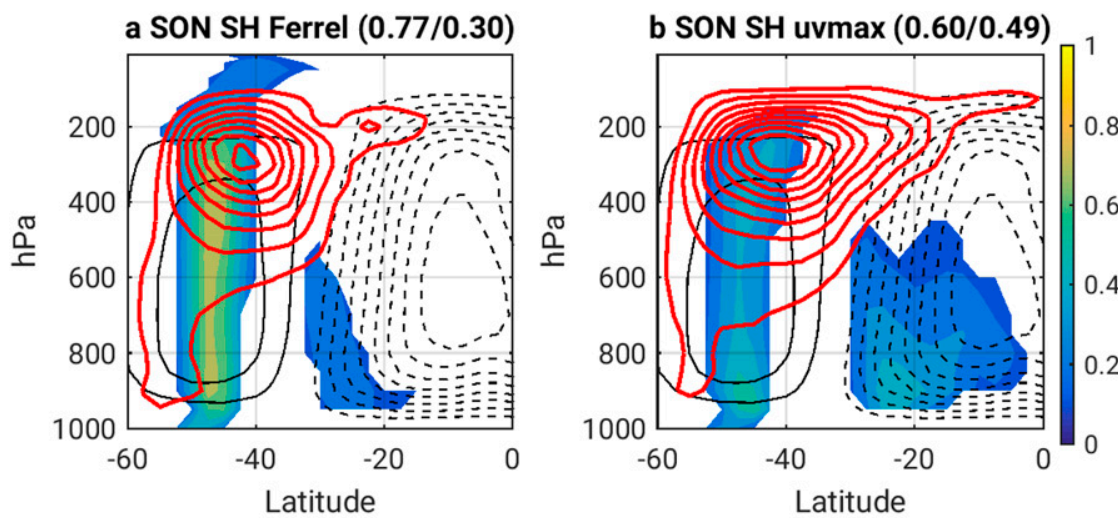

c SON SH Ferrel. Zonal wind
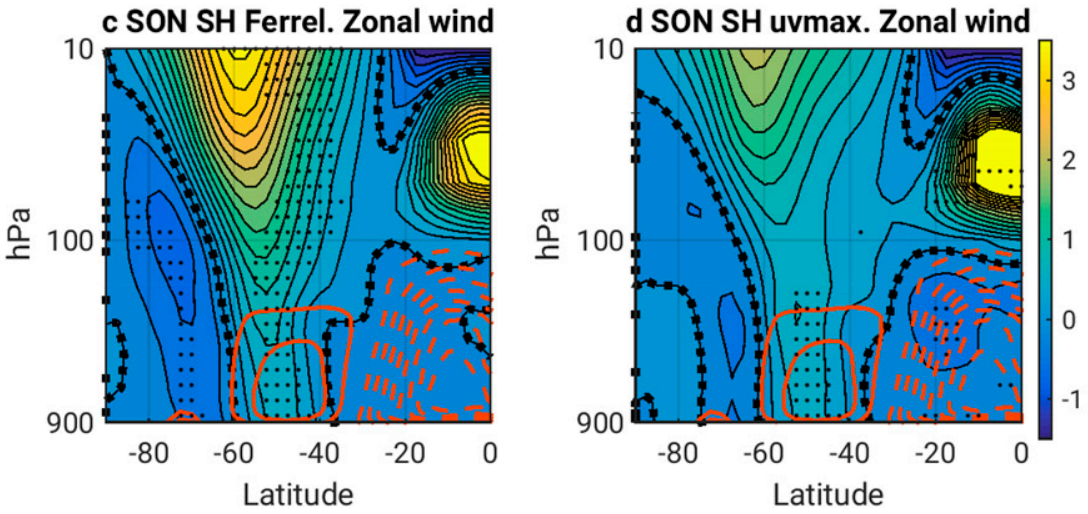

FIG. 3. (a),(b) For the SH during austral spring (SON) and the (a) Ferrel cell index or the (b) uvmax index: climatological-mean streamfunction (black contours, with negative contours dashed; contour interval: $1.5 \times 10^{10} \mathrm{~kg} \mathrm{~s}^{-1}$ ), fraction of streamfunction variance $r^{2}$ explained by the index (shading; only values significant at the $95 \%$ level are shown), and regressed meridional eddy momentum flux (red contours; contour interval: $0.5 \mathrm{~m} \mathrm{~s}^{-1}$ ). The numbers in parentheses in panel titles list the maximum explained variance over the Ferrel and Hadley cells, respectively. (c),(d) As in (a),(b), but for the zonal-wind regression (shading in $\mathrm{m} \mathrm{s}^{-1}$, with the zero wind contour in the black thick-dotted line; regions exceeding the $95 \%$ significance level are shown stippled). The orange contours show the climatological streamfunction. 
equilibrate the eddy forcing $S$ over the region of subtropical subsidence, where $\bar{v}$ vanishes and vertical shear is large. The vertical eddy momentum divergence can also be a significant contribution to $S$ over this region. For illustration, Fig. $2 \mathrm{f}$ shows the regression of the net eddy forcing $S$ (for all eddies) on the Ferrel cell index during DJF, which should be compared with the meridional divergence regressions previously discussed in Fig. 2d. It is remarkable that the (anomalous) full eddy forcing vanishes at the level of the subtropical jet, as the meridional synoptic divergence (blue contours in Fig. 2d) is very nearly compensated by vertical planetary convergence (not shown). This is as required by the weak MMC advection (both horizontal and vertical) over this region.

For a perturbed flow, we linearize the first term in the momentum balance following $\mathrm{C} 07$ :

$$
(f+\bar{\xi}) \delta \bar{v}+\bar{v} \delta \bar{\xi}-\delta\left(\bar{\omega} \bar{u}_{p}\right)=\delta S .
$$

We refer to the three terms on the left-hand side as the MMC response, the vorticity response, and the vertical advection response. As our main interest is on the latitudes of poleward flow, where vertical advection is small, no effort is made to linearize this last term.

To provide context for the results presented below, we discuss how this anomalous balance may be satisfied in the $\mathrm{NH}^{1}$ under different scenarios. First, when an anomalous eddy drag $\delta S>0$ decelerates the tropical upper troposphere, the perturbed equilibrium requires enhanced acceleration by the mean flow advection. This may occur through an intensification of the Hadley circulation $\delta \bar{v}>0$ with fixed angular momentum gradients (note that $f+\bar{\xi}=-d M / d y>0$, where $M$ is absolute angular momentum), or through an intensification of these gradients when $\delta \bar{\xi}>0$. The latter is associated with a reduction in the anticyclonic relative vorticity $\bar{\xi}<0$ and a slowdown of the subtropical jet, and with an increase in the absolute vorticity (or angular momentum gradient) $f+\bar{\xi}$. On the other hand, the Hadley cell may also intensify $(\delta \bar{v}>0)$ with no eddy drag, for instance, forced by changes in tropical heating. In these circumstances, the Hadley cell intensification must be accompanied by a reduction in the angular momentum gradient $f+\bar{\xi}$ so as to keep the mean flow advection unchanged. The reduction in $f+\bar{\xi}$ is associated with increased negative $\bar{\xi}$ (and enhanced subtropical winds), compensating the planetary vorticity $f$, with the limit $f+\bar{\xi}=0$ corresponding to angular momentum conservation. Finally,

\footnotetext{
${ }^{1}$ Similar arguments hold in the $\mathrm{SH}$, with the signs of $f, \bar{\xi}$, and $\bar{v}$ reversed.
}

it is also possible that changes in vertical advection compensate the anomalous meridional advection in the absence of eddies, with two interesting limits. With fixed circulation, when $\bar{v} \delta \bar{\xi}-\bar{\omega} \delta \bar{u}_{p}=0$, the anomalous mean flow advection vanishes because changes in angular momentum are constant along the mean streamlines: $J(\bar{\psi}, \delta M)=0$. Similarly, with fixed angular momentum gradients, there is no mean flow advection when $(f+\bar{\xi}) \delta \bar{v}-\bar{u}_{p} \delta \bar{\omega}=0$, as the anomalous MMC is then directed along angular momentum isocontours: $J(\delta \bar{\psi}, M)=0$. We expect this last constraint to be more relevant in the subtropics, where the Rossby number is small, and changes in angular momentum gradients are less important than changes in the circulation for the zonal momentum balance. Since $M$ decreases poleward and downward, the compensation of the two advection terms is typically associated with the equatorward sloping of the subsiding flow.

\section{a. Northern Hemisphere winter}

Figure 4 (left) describes the northern winter anomalous momentum balance associated with enhanced momentum forcing, as depicted by regression on the uvmax index. The upper panel shows the anomalous eddy momentum divergence $\delta S$ (shading) and the meridional contributions by synoptic, extratropical planetary, and intertropical planetary eddies (blue, maroon, and magenta contours, respectively, as in Fig. 2). As discussed above, the patterns of meridional and total eddy divergence differ significantly in the subtropics because of the contribution by the vertical eddy momentum flux. The middle and bottom panels in Fig. 4 show the MMC and vorticity responses (the vertical advection term is only important in the subtropics and has not been included), with the net eddy divergence contours overlaid to facilitate comparison with the eddy forcing., As expected based on the small Rossby numbers in the subtropics $(\mathrm{Ro}=|\bar{\xi}| / f<0.1)$, the anomalous eddy forcing is mainly balanced by the MMC response over this region, with an additional contribution by the vertical advection response (not shown). In contrast, over the latitudes of meridional outflow $\left(10^{\circ}-20^{\circ}\right)$, the response is qualitatively different in the mid- and upper troposphere. Consistent with Fig. 1, we can see that the bulk of the MMC response is found below the level of maximum eddy forcing, while the vorticity response dominates at upper levels. This vorticity response is dynamically associated with the deceleration of the upper-tropospheric westerlies by the planetary momentum divergence (Fig. 5a), which decreases the meridional wind shear and anticyclonic relative vorticity and increases the absolute vorticity.

We can understand the vertical structure of the response by noting that the mean meridional flow $\bar{v}$ is 

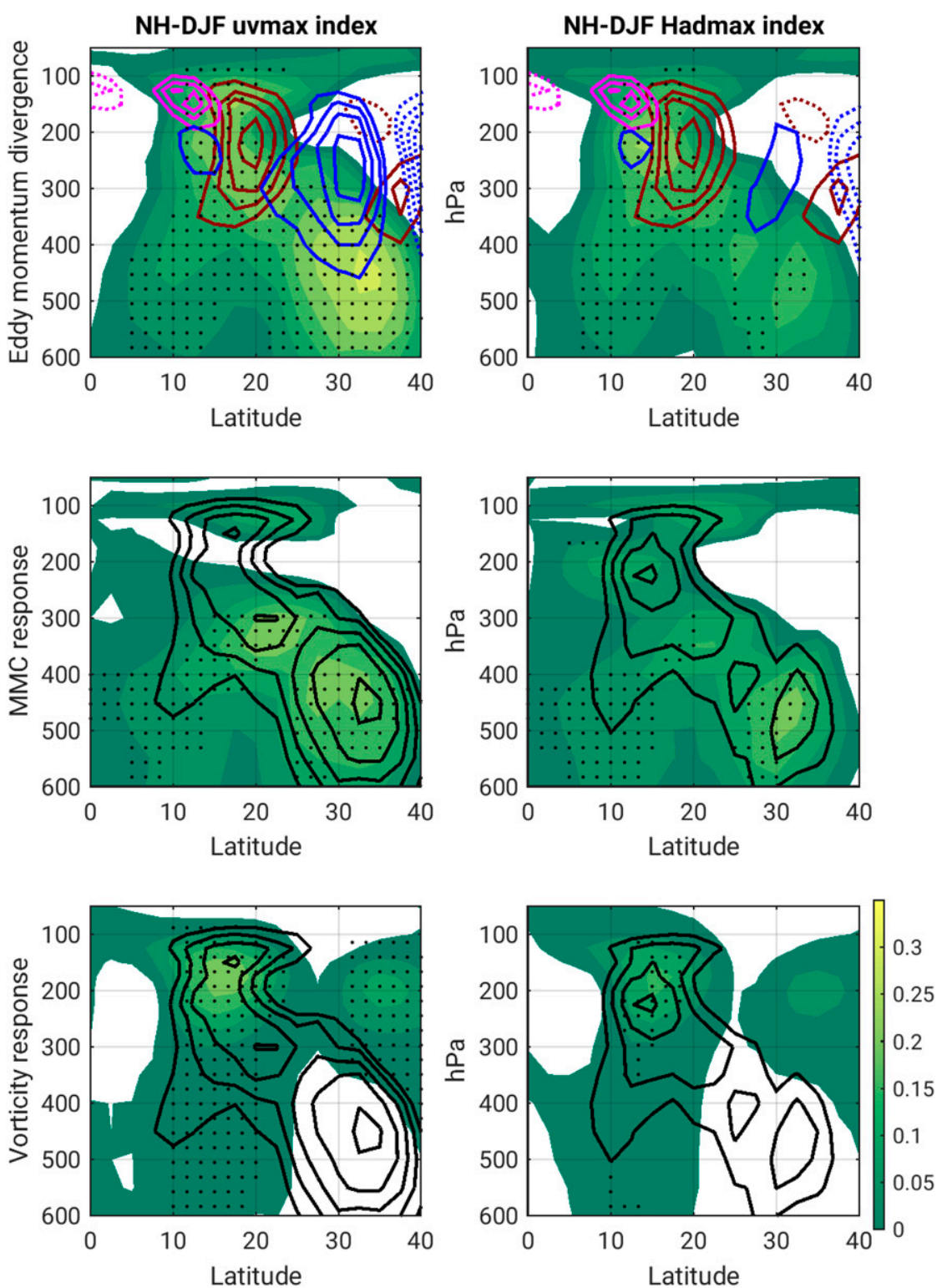

FIG. 4. During boreal winter, regression on the (left) uvmax and (right) Hadmax indices of (top) net eddy momentum divergence $S$ (shading) and decomposition of its meridional component into synoptic, extratropical planetary, and intertropical planetary contributions (contours as in Fig. 2e), (middle) MMC response, with the net eddy divergence overlaid for reference in black contours, and (bottom) as in the top and middle panels, but for the vorticity response. Only positive values are shaded (units in $\mathrm{m} \mathrm{s}^{-1} \mathrm{day}^{-1}$ ), with regions exceeding the $95 \%$ significance level shown stippled.

strongly peaked at upper levels (Fig. 1c), much more so than the mean absolute vorticity $f+\bar{\xi}$ or the perturbed eddy forcing $\delta S$. Using hydrostatic balance, the vertical gradient of $\delta \bar{\xi}$ is proportional to the horizontal Laplacian of temperature $\nabla^{2} \delta T$, which implies that $\delta \bar{\xi}$ must vary slowly in the vertical when temperature gradients are weak in the tropics (note the slow vertical decay of the wind anomalies in Fig. 5a). As a result, the vorticity response $\bar{v} \delta \bar{\xi}$ is strongly confined at upper levels, where weak $\delta \bar{\xi}$ corrections suffice to balance the eddy forcing (note the small magnitude of the associated wind anomalies in Fig. 5). Away from this region of strong meridional flow, the vorticity response decays sharply and the response to $\delta S$ must be dominated by the MMC response. Note that the vorticity response is found over regions with larger Rossby numbers than the MMC response, with the 

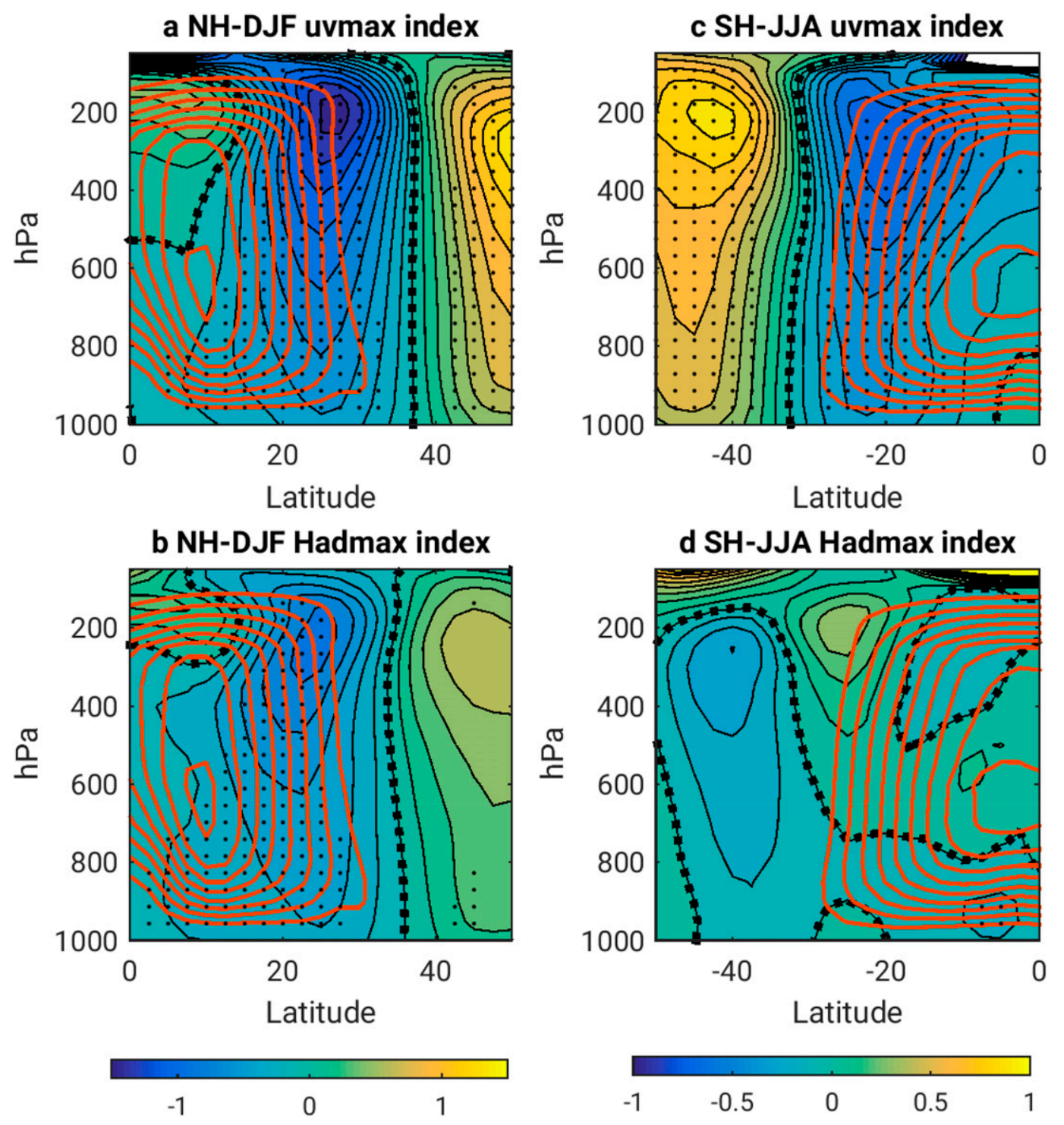

FIG. 5. Zonal-wind regression (shading in $\mathrm{m} \mathrm{s}^{-1}$, with the zero wind contour in the black thick-dotted line; regions exceeding the $95 \%$ significance level are shown stippled) on the (a),(c) uvmax index and the (b), (d) Hadmax index during the (left) boreal and (right) austral winters and climatological Hadley cell (orange contours; contour interval: $\left.2.5 \times 10^{10} \mathrm{~kg} \mathrm{~s}^{-1}\right)$.

transition occurring around $\mathrm{Ro}=0.35$. However, differences in the Rossby number alone cannot explain the abrupt transition in the response because, like zonal wind, Ro changes slowly with pressure (not shown).

The right panels of Fig. 4 display a similar analysis for the perturbed momentum balance associated with strengthening of the northern winter Hadley cell, now based on regression on the Hadmax index. The main difference from the previous case is the near disappearance of the synoptic eddy divergence, consistent with the notion that only planetary eddies can affect the maximum Hadley overturning. The planetary eddy forcing is balanced by both the MMC and the vorticity responses, the latter again dominating at upper levels. Consistent with eddy driving of the $\mathrm{NH}$ Hadley cell variability, the zonal-wind regression on the Hadmax index is similar (but weaker) to the uvmax regression, both showing a deceleration of the subtropical jet by the enhanced eddy momentum fluxes (Figs. 5a,b).

\section{b. Southern Hemisphere winter}

In contrast with the $\mathrm{NH}$, the perturbed tropical momentum balance in the $\mathrm{SH}$ during austral winter (JJA) is qualitatively different when regressed onto momentum 

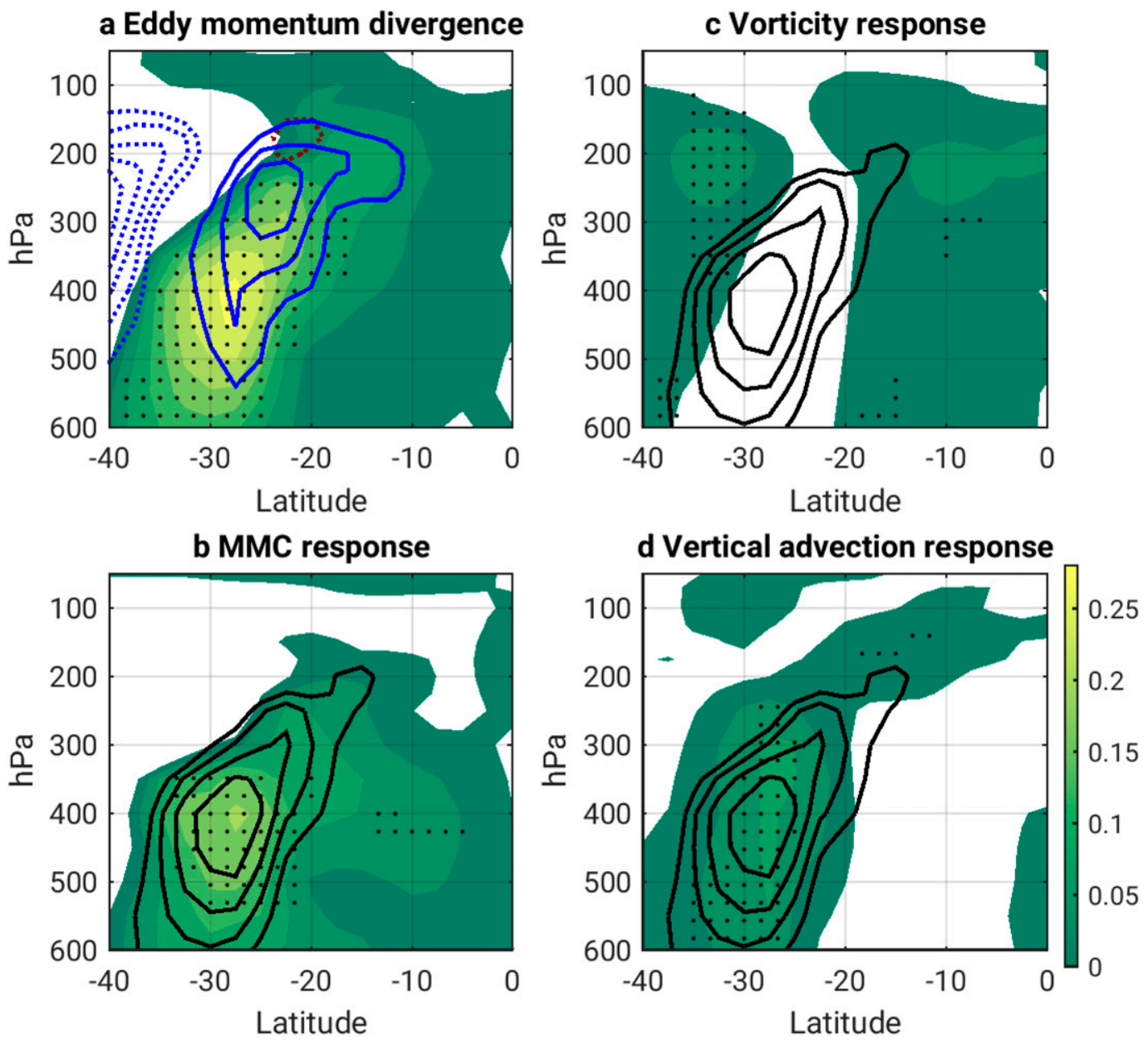

FIG. 6. (a)-(c) As in the left panels of Fig. 4, but for the SH during austral winter: regression of the net eddy forcing, MMC response, and vorticity response on the uvmax index, with the eddy divergence overlaid in contours. The stippling marks the $95 \%$ significance level. (d) As in (a)-(c), but for the vertical advection response

forcing (uvmax regression; Fig. 6) and onto Hadley cell strength (Hadmax regression; Fig. 7).

The response to the eddy momentum forcing (Fig. 6) is dominated by the MMC response in the subtropics, as the eddy momentum divergence (now purely synoptic) penetrates little into the tropics. The vertical advection response is also important over this region. The vorticity response is still the dominant term in the tropical upper troposphere, but its magnitude is much less than in the $\mathrm{NH}$ (and not significant) because of the weak eddy penetration into that region.

The Hadmax regression (Fig. 7) is qualitatively different from all previous analyses, as the eddy momentum divergence is now very small over the tropics. As noted above, with $\delta S \approx 0$, Eq. (3) requires the positive MMC forcing in the upper troposphere associated with the Hadley cell strengthening (Fig. 7b) to be compensated by a negative vorticity forcing $\bar{v} \delta \bar{\xi}<0$, consistent with Fig. 7c. This implies that relative vorticity must increase (note that $\bar{v}<0$ in the $\mathrm{SH}$ ), as the upper-troposphere westerlies spin up (Fig. 5d) and the circulation becomes closer to the angular momentum-conserving limit $f+\bar{\xi}=0$ in contrast to all previous cases. However, note that the vorticity signal is weak (note the reduced scale compared to the previous plots) and not significant at the $95 \%$ level. The same is true for the zonal-wind regression in Fig. 5d.

On the other hand, there is a significant positive forcing by the vertical advection term between $20^{\circ}$ and $30^{\circ}$ below $200 \mathrm{hPa}$, which tends to balance the negative forcing by the meridional advection term over the same region. As noted above, the compensation of the two mean advection terms is associated with the alignment of the anomalous circulation along the angular momentum isocontours, while the sign of these terms is consistent with the equatorward drift of the descending 

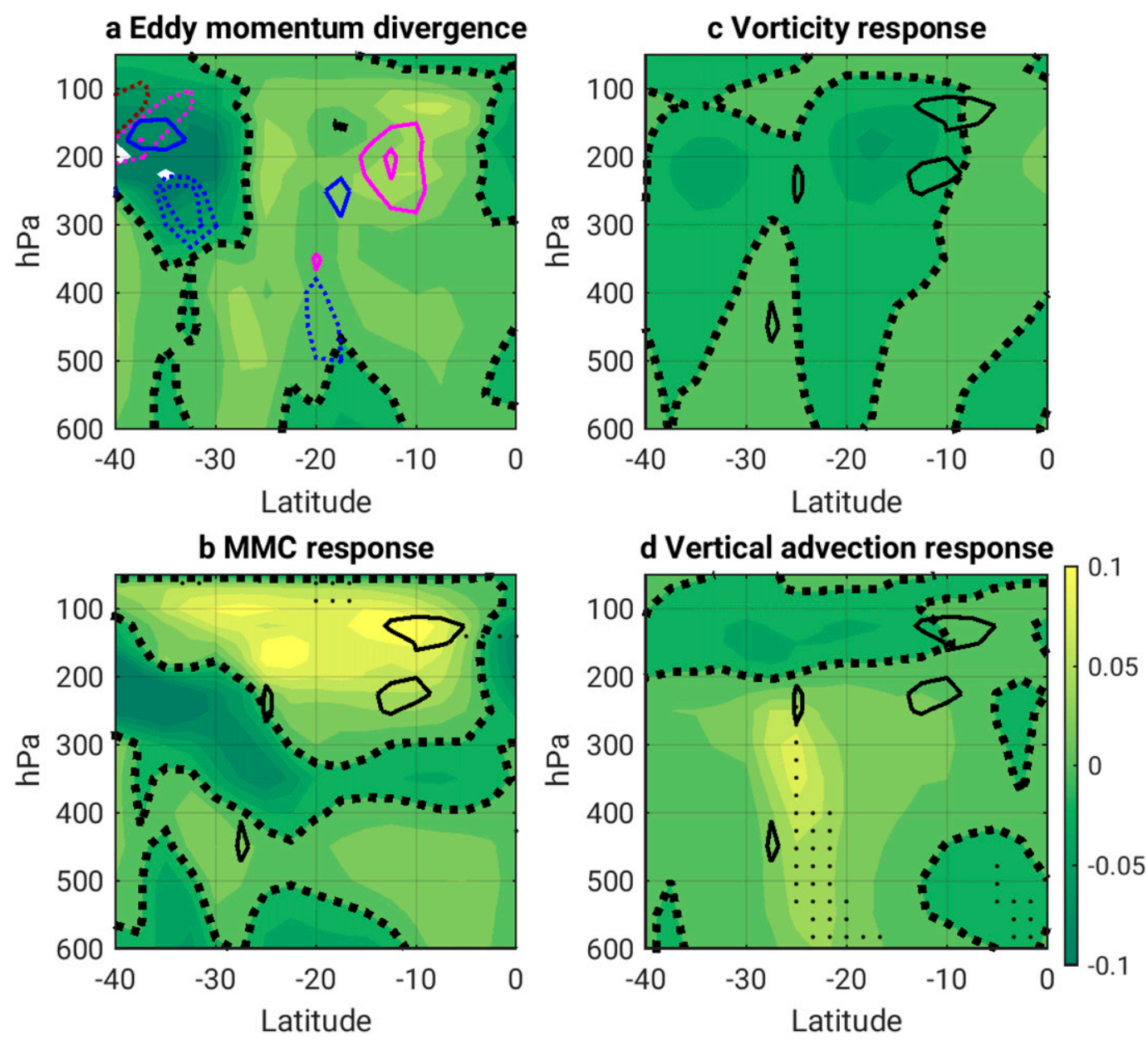

FIG. 7. As in Fig. 6, but for the regressions on the Hadmax index. Note that both positive and negative values are shaded now (the black thick-dotted contour shows the zero line), and the reduced color scale compared to the previous plots. The contour interval for the eddy divergence contours is also halved.

flow. This is illustrated by the streamfunction regression on Hadmax (Fig. 8a), which displays the characteristic slanted structure of the eddy-free solution (Held and Hou 1980). We discuss this structure in more detail in the next section. It is also interesting that there is little spatial overlap between the Hadmax and uvmax streamfunction regressions in the Southern Hemisphere, as each index captures the variability of a different region of the cell (Figs. 8a,b), suggesting that the two modes of variability are orthogonal. In contrast, the northern winter Hadmax and uvmax regressions have a more similar structure (Figs. 8c,d), though the former cell is deeper for reasons discussed above.

\section{c. MMC momentum transport and boundary layer winds}

Consistent with the argument that the anomalous southern winter Hadley cell approximately conserves angular momentum, changes in Hadmax during JJA have very little impact on the vertically integrated momentum balance. To see this, we compare the regressed surface-wind pattern for all the cases discussed in the previous section (Fig. 8, bottom panels). Because the anomalous frictional force is proportional to the surfacewind anomalies, the magnitude of this regression gives an indication of the net column momentum forcing. We can see that although the variability in the northern and southern Hadley cells has comparable strength during their respective winters (Fig. 8, top panels), the variability of the southern cell is associated with a much weaker near-surface wind response (cf. the blue lines in the bottom panels of Fig. 8).

In the quasigeostrophic (QG) approximation, the upper-level momentum divergence $S$ is balanced by the Coriolis torque acting on the meridional poleward flow $S \approx f V$, while the Coriolis torque acting on the return flow of this circulation produces the same acceleration $S$ over the boundary layer, where it is equilibrated by 

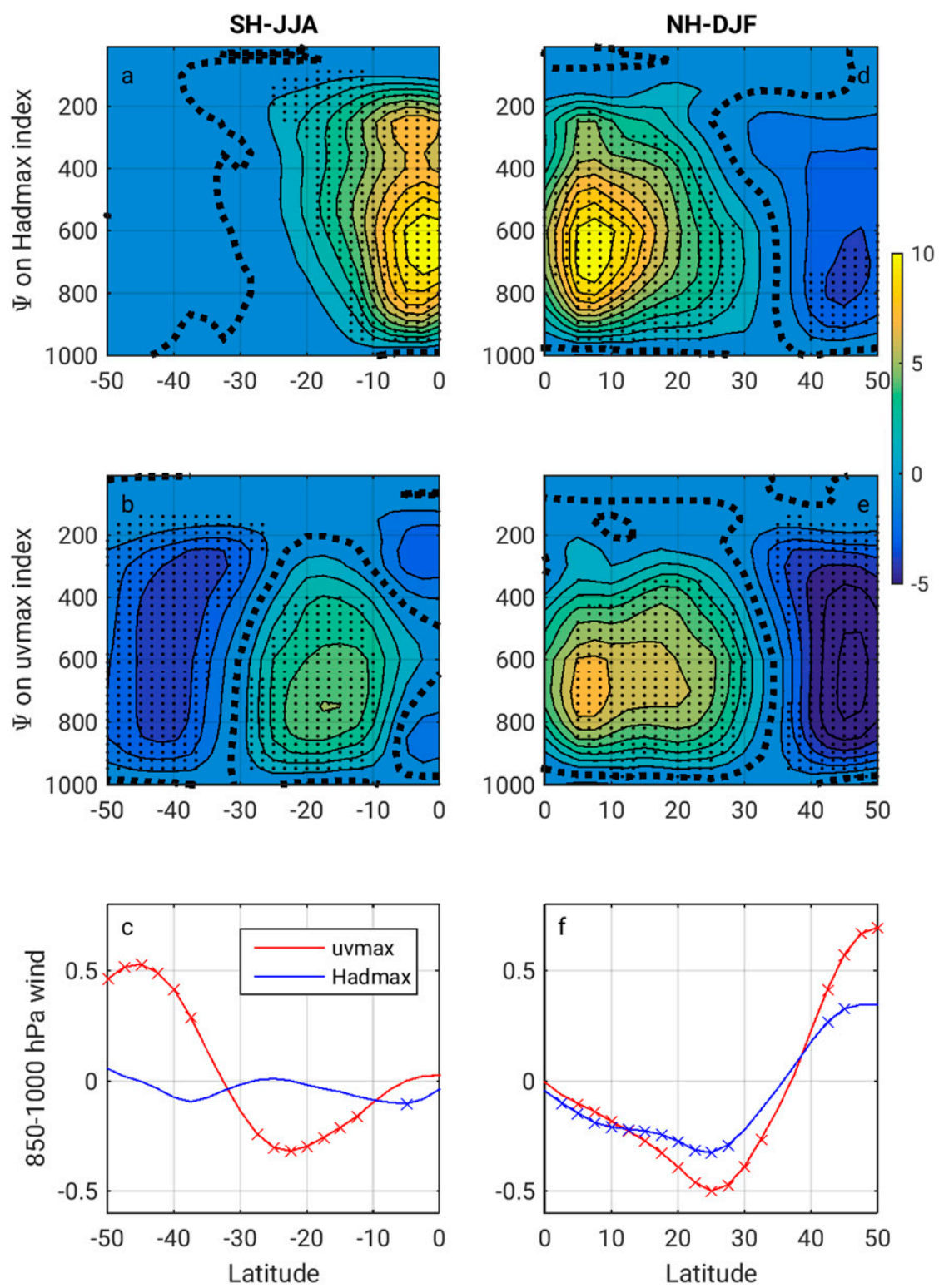

FIG. 8. For (left) the SH during austral winter and (right) the NH during boreal winter: (top) Regression of the mass streamfunction on the Hadmax index (in $10^{9} \mathrm{~kg} \mathrm{~s}^{-1}$; the zero contour is shown with the black thick-dotted line), (middle) regression of the mass streamfunction on the uvmax index, and (bottom) regression of the mean lower-troposphere zonal wind on the uvmax (red) and Hadmax (blue) indices. The $95 \%$ confidence level is shown with stippling in the top four panels and with crosses in the bottom two.

friction $-f V \approx$ Fric. Thus, in the QG approximation, the MMC only transports momentum vertically, and upper-tropospheric eddy drags are locally balanced by friction at the same latitude: $S+$ Fric $\approx 0$. As a result, no surface-wind signal would be expected in the absence of eddy drag under QG scaling. However, the QG approximation does not work well in the deep tropics: as the poleward momentum transport by the $\mathrm{MMC}$ is also important in this region, the anomalous surface wind may penetrate deeper into the tropics than the eddy momentum forcing (cf. the red line in Fig. $8 \mathrm{c}$ and the blue line in Fig. 2a for the SH uvmax regressions).

Vertically integrated, the Hadley cell transports momentum from the tropics to the subtropics. Over the latitudes of meridional flow, the Hadley cell produces an upper-level acceleration but a net column deceleration given by 


$$
\mathrm{F}_{\mathrm{MMC}}=\int(f+\bar{\xi}) \bar{v} d p=V\left(\xi_{u}-\xi_{l}\right) \approx V \xi_{u}<0,
$$

where $V$ is the net poleward mass flow, and $\xi_{u}$ and $\xi_{l}$ are the mean relative vorticity over the regions of poleward and equatorward flow, respectively (the latter is usually negligible). Because $V$ and $\xi_{u}$ have opposite signs (positive and negative, respectively, in the $\mathrm{NH}$, and the reverse in the $\mathrm{SH}$ ), this produces a net deceleration over most tropical latitudes. This deceleration is compensated by acceleration in the subtropics by the vertical advection term $\mathrm{F}_{\mathrm{MMC}}=-\int \bar{\omega} \bar{u}_{p} d p>0$ (note that the net MMC momentum forcing must vanish when integrated over a closed streamline).

However, this MMC transport requires that some drag decelerates the westerly flow (otherwise $\xi_{u}$ and $\xi_{l}$ would be equal and $\bar{u}_{p}$ would vanish, and there would be no column momentum forcing in tropics or subtropics). As illustrated by the sketch in Fig. 9, the meridional MMC momentum transport across a latitude $\phi_{0}$ depends on the net drag exerted on the circulation poleward of that latitude, which determines the zonal-wind difference between upper and lower levels $U_{u}-U_{l}$. In the absence of eddy drag (angular momentum-conserving limit), the drag on the mean flow is entirely frictional (which requires subtropical westerlies) and all the meridional advection of angular momentum occurs inside the boundary layer. Assuming that friction is strong enough to make $U_{l} \ll U_{u}$, this limit provides the maximum $U_{u}-U_{l}$ difference, and hence the maximum MMC meridional momentum transport over those latitudes where the return flow occurs within the boundary layer. The magnitude of this frictional transport is, however, severely reduced when the circulation tilts equatorward as it descends and the return flow enters the boundary layer at low latitudes as in Fig. 8a. If we estimate the MMC momentum transport for the eddy-free circulation to have order $V U_{\mathrm{AMC}}\left(\phi_{\mathrm{BL}}\right)$, where $U_{\mathrm{AMC}}(\phi)$ is the angular momentum-conserving wind and $\phi_{\mathrm{BL}}$ is the latitude at which the return flow enters the boundary layer, this transport can be quite small when $\phi_{\mathrm{BL}}$ is close to the equator.

This frictional limit is obviously not very relevant in the atmosphere, as the climatological surface wind vanishes near the Hadley cell edge. This implies that the vertical advective acceleration by the Hadley cell in the subtropics $\mathrm{F}_{\mathrm{MMC}}=-\int \bar{\omega} \bar{u}_{p} d p>0$ is not balanced by friction but by the eddy drag $S$ over those latitudes. The importance of the eddy drag for driving the MMC momentum transport is further demonstrated by the structure of the Hadmax regressions in Fig. 8. These show that the anomalous southern Hadley cell intensification is nearly inviscid during austral winter in the absence of

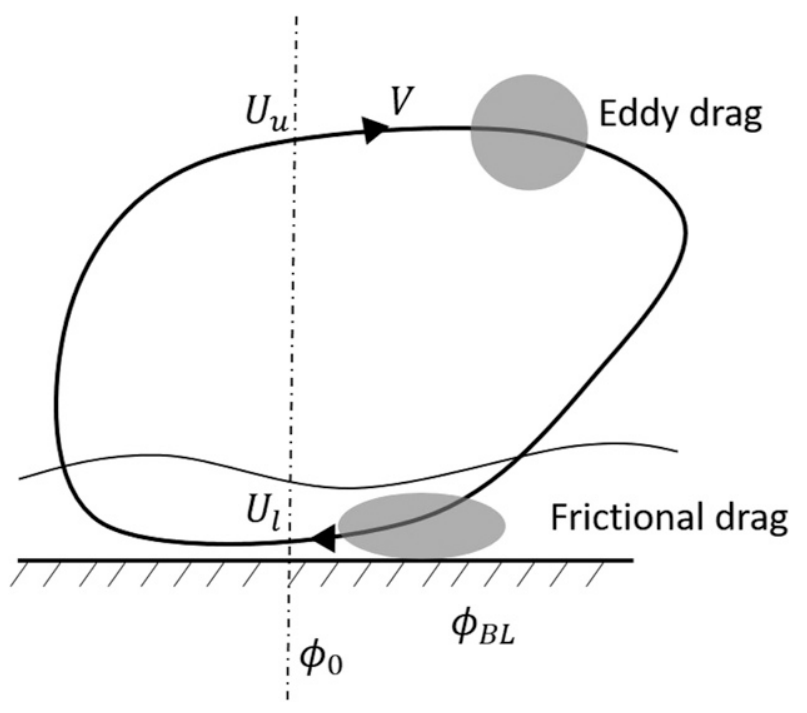

FIG. 9. Sketch illustrating how the meridional MMC momentum flux $V\left(U_{u}-U_{l}\right)$ across a latitude $\phi_{0}$ depends on the net (eddy plus frictional) drag acting on the Hadley flow poleward of that latitude. The frictional drag decreases as the circulation tilts equatorward and the latitude $\phi_{\mathrm{BL}}$ at which the return flow enters the boundary layer is reduced.

eddy drag. The subsiding flow tilts equatorward and enters the boundary layer at fairly low latitudes, so that the anomalous boundary layer winds are very small as noted above.

\section{Discussion}

This work has analyzed the interannual covariability between the Hadley and Ferrel cells for all seasons. During DJF our results are consistent with $\mathrm{C} 07$, who noted that Hadley cell variability in both hemispheres was partly eddy-driven during this season. Also consistent with that work, the northern winter correlation is driven by extratropical planetary eddies while the southern summer correlation is driven by synoptic eddies (not shown). However, no significant correlation was found between the extratropical circulation and the maximum Hadley overturning in any other season. The lack of correlation appears to be due to the weak eddy penetration into the deep tropics, as proposed by Bordoni and Schneider (2008), for the southern Hadley cell during boreal summer, a season with uppertropospheric easterlies in the deep tropics. Our results suggest that eddy penetration in the deep tropics is also weak during other seasons because only planetary eddies are able to propagate significantly past the subtropical jet core. Although the maximum Hadley overturning is only weakly correlated with Ferrel cell variability during most seasons, the subtropical part of 

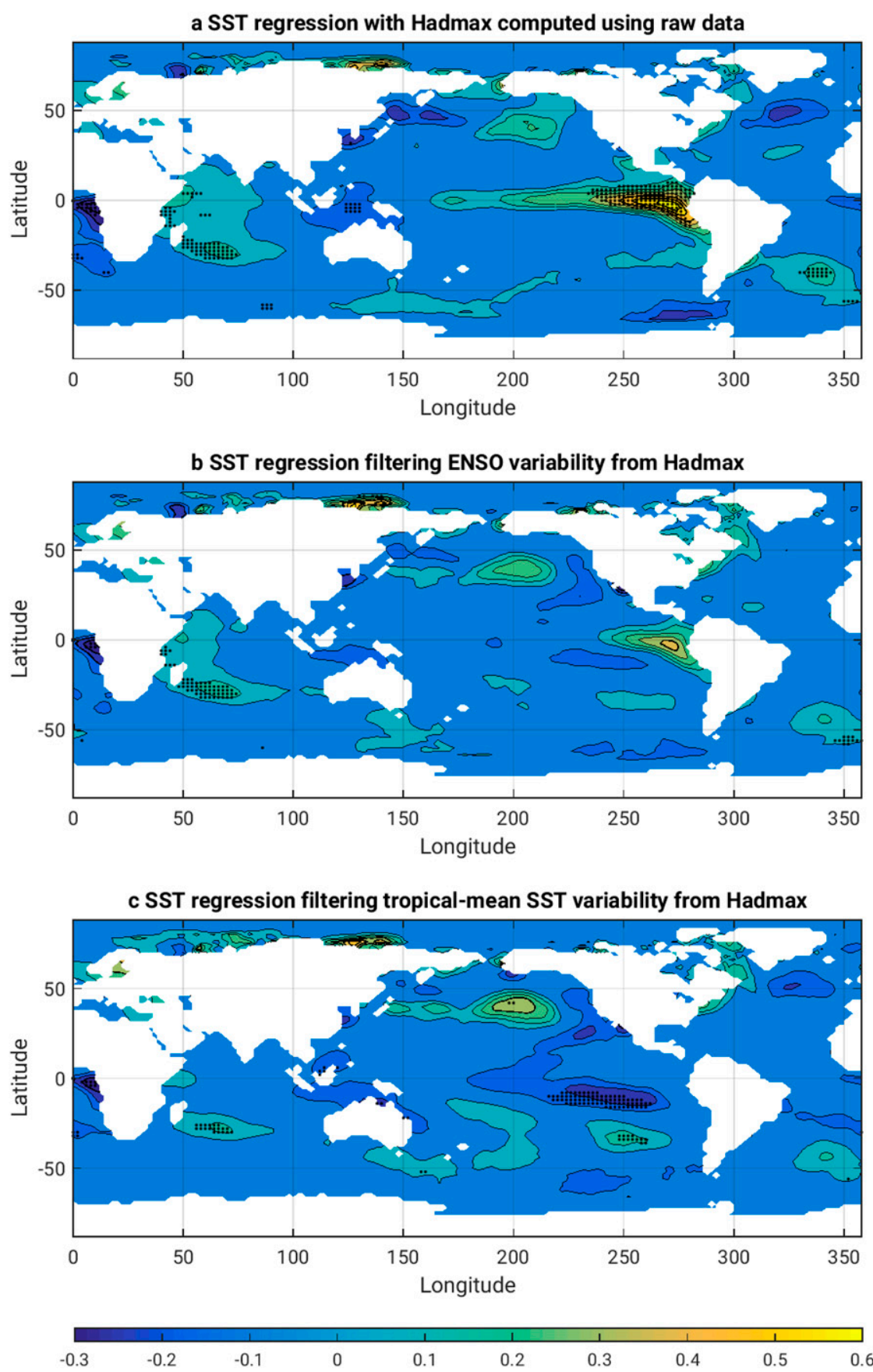

FIG. 10. SST regression on the SH Hadmax index during austral winter (contour interval: $0.075^{\circ} \mathrm{C}$; the zero contour is not plotted) when this index is computed (a) using raw data; (b) filtering out ENSO-3.4 variability; and (c) filtering out the variability associated with the tropical-mean SST $(|\phi|<5 \mathrm{deg})$. The stippling marks the 95\% significance level.

the Hadley cell is significantly correlated with the extratropical eddy momentum flux for all seasons.

The southern summer circulation provides the best example of an eddy-driven Hadley cell, as the thermally driven circulation is weak in the summer hemisphere (Lindzen and Hou 1988) and extratropical eddies are only slightly weaker during southern summer than during southern winter. Indeed, Fig. 1a shows that the mean Hadley and Ferrel cells have comparable intensities during this season (the Hadley cell is a bit stronger, which could simply reflect the smaller values of the Coriolis parameter in the tropics). In the opposite 

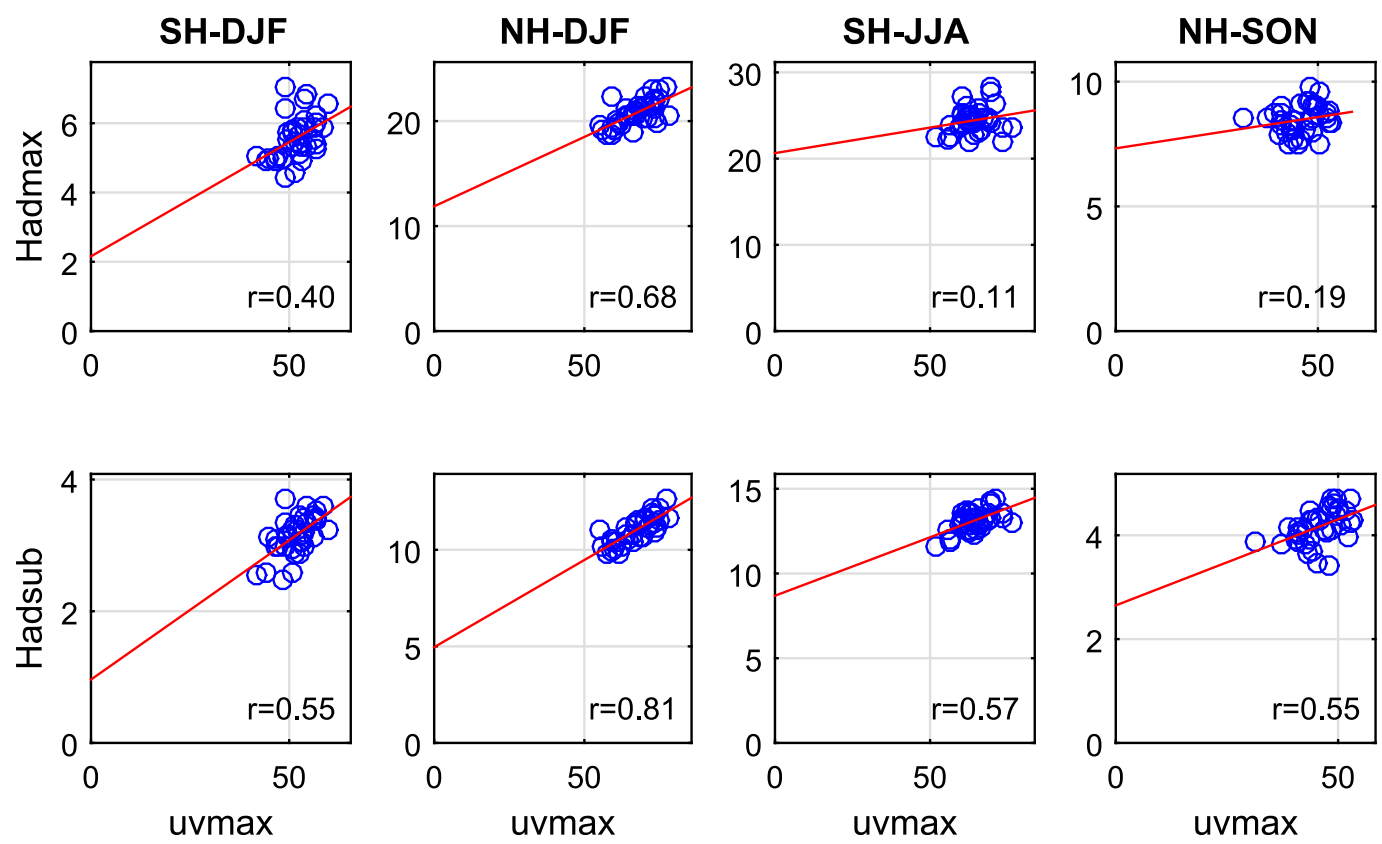

FIG. 11. Scatterplots between the (top) Hadmax or (bottom) Hadsub Hadley indices and the index uvmax measuring the subtropical momentum forcing for select seasons. The red lines show the best linear fits and the text shows the detrended correlations (as in Table 2).

regime, we have shown that the variability of the southern winter Hadley cell is governed by nearly angular momentum-conserving dynamics (Held and Hou 1980), as the core of this circulation is effectively isolated from the influence of extratropical eddies.

In this limit we would expect Hadley cell variability to be driven by changes in tropical heating, but we have failed to find convincing evidence of SST driving. Figure 10a shows the JJA SST regression on the southern Hadmax index when this index is computed using raw data, and Fig. 10b shows the same when ENSO-3.4 variability is filtered out from the data. This eliminates the central Pacific SST anomalies, but some tropical Pacific anomalies still remain confined to the eastern Pacific. However, ENSO filtering only reduces the variance of the Hadmax variability by about $10 \%$. We have tried to filter out the tropical SST variability using other indices, standard and nonstandard, with similar results. For example, Fig. 10c shows the SST regression on the southern Hadmax index when the detrended tropicalbelt SST variability (mean SST averaged within $5^{\circ}$ of the equator) is subtracted, which also eliminates the eastern Pacific signal. The reduction in Hadmax variance is only slightly larger than with ENSO filtering.

Although our work has focused on the coupled HadleyFerrel variability, we note that a significant correlation between both Hadley cells was also found during MAM. The SON Hadley-Hadley correlation (0.36) does not exceed the $99 \%$ confidence level but is still significant at a
93\% level. A cursory inspection of the SST regression on the Hadley indices during MAM did not reveal any obvious pattern (not shown), but this needs to be investigated more carefully. It is possible that both Hadley circulations respond to the same tropical planetary wave forcing, which is known to impact Hadley cell variability (Dima et al. 2005; Shaw 2014). Alternatively, the equinoctial HadleyHadley correlation might be driven from the stratosphere, for instance, modulated by changes in the QBO phase (note the significant correlation between tropical stratospheric zonal wind and subtropical eddy momentum flux during SON in Fig. 3d). We plan to analyze the HadleyHadley covariability in more detail in a future study.

As noted in the introduction, the processes forcing the climatological Hadley cell are not necessarily the same driving its internal variability. Although we cannot really address the first question using the observed internal

TABLE 3. Estimate of the eddy-driven component of the climatological Hadley cell based on the linear regression analysis (see text for details).

\begin{tabular}{lccccc}
\hline & \multicolumn{2}{c}{ Southern Hemisphere } & & \multicolumn{2}{c}{ Northern Hemisphere } \\
\cline { 2 - 3 } \cline { 5 - 6 } & Hadmax & Hadsub & & Hadmax & Hadsub \\
\hline DJF & $61 \%$ & $70 \%$ & & $43 \%$ & $55 \%$ \\
MAM & $25 \%$ & $63 \%$ & & $24 \%$ & $43 \%$ \\
JJA & $16 \%$ & $34 \%$ & & $39 \%$ & $50 \%$ \\
SON & $58 \%$ & $63 \%$ & & $14 \%$ & $36 \%$ \\
ANN & $53 \%$ & $70 \%$ & $27 \%$ & $63 \%$ \\
\hline
\end{tabular}


variability, we can infer a tentative estimate for the eddy impact on the mean circulation from the linear regression of the relevant indices (Fig. 11, top panels). Using the intercept of this regression as a measure of the eddy-free circulation, differences with this intercept provide an estimate of the impact of the eddy driving for the observed circulation. By this measure, the eddies explain about $61 \%$ of the climatological summer Hadley cell in the SH, but only $16 \%$ of its winter counterpart. As expected, these percentages increase when only the subtropical Hadley cell index is considered (to $70 \%$ and $34 \%$, respectively; see bottom panels of Fig. 11). Table 3 shows the results of this analysis for all seasons.

In conclusion, we have shown that extratropical eddies play an important role for the variability of the outermost Hadley cell in all seasons but are only able to affect the Hadley cell core during DJF in both hemispheres, as was already found by $\mathrm{C} 07$.

Acknowledgments. We thank the three anonymous reviewers for their excellent reviews providing new insight and very detailed comments that improved the clarity of the manuscript. One of the reviewers alerted us to the important role played by the vertical eddy momentum divergence in the subtropics. NOAA-ERSST data was provided by the NOAA/OAR/ESRL PSD, Boulder, Colorado, from their website at http://www.esrl. noaa.gov/psd/. We thank NOAA for this data, and the European Centre for Medium-Range Weather Forecasts for the atmospheric reanalysis data. P. Z.-G. acknowledges financial support by Grant CGL2015-72259-EXP by the Ministry of Economy and Innovation of Spain.

\section{REFERENCES}

Bordoni, S., and T. Schneider, 2008: Monsoons as eddy-mediated regime transitions of the tropical overturning circulation. Nat. Geosci., 1, 515-519, https://doi.org/10.1038/ngeo248.

Caballero, R., 2007: Role of eddies in the interannual variability of Hadley cell strength. Geophys. Res. Lett., 34, L22705, https:// doi.org/10.1029/2007GL030971.

Ceppi, P., and D. L. Hartmann, 2013: On the speed of the eddydriven jet and the width of the Hadley cell in the Southern Hemisphere. J. Climate, 26, 3450-3465, https://doi.org/ 10.1175/JCLI-D-12-00414.1.

Chang, E. K. M., 1996: Mean meridional circulation driven by eddy forcings of different timescales. J. Atmos. Sci., 53, 113-125, https://doi.org/10.1175/1520-0469(1996)053<0113: MMCDBE $>2.0 . \mathrm{CO} ; 2$.

Davis, N., and T. Birner, 2017: On the discrepancies in tropical belt expansion between reanalyses and climate models and among tropical belt width metrics. J. Climate, 30, 1211-1231, https:// doi.org/10.1175/JCLI-D-16-0371.1.

Davis, S. M., and K. H. Rosenlof, 2012: A multidiagnostic intercomparison of tropical-width time series using reanalyses and satellite observations. J. Climate, 25, 1061-1078, https:// doi.org/10.1175/JCLI-D-11-00127.1.

Dee, D. P., and Coauthors, 2011: The ERA-Interim reanalysis: Configuration and performance of the data assimilation system. Quart. J. Roy. Meteor. Soc., 137, 553-597, https://doi.org/ 10.1002/qj.828.

Dima, I. M., J. M. Wallace, and I. Kraucunas, 2005: Tropical zonal momentum balance in the NCEP reanalyses. J. Atmos. Sci., 62 , 2499-2513, https://doi.org/10.1175/JAS3486.1.

Held, I. M., and A. Y. Hou, 1980: Nonlinear axially symmetric circulations in a nearly inviscid atmosphere. J. Atmos. Sci., 37, 515-533, https://doi.org/10.1175/1520-0469(1980)037<0515: NASCIA $>2.0 . \mathrm{CO} ; 2$.

Huang, B., and Coauthors, 2015: Extended Reconstructed Sea Surface Temperature version 4 (ERSST.v4). Part I: Upgrades and intercomparisons. J. Climate, 28, 911-930, https://doi.org/ 10.1175/JCLI-D-14-00006.1.

Kang, S. M., and L. M. Polvani, 2011: The interannual relationship between the latitude of the eddy-driven jet and the edge of the Hadley cell. J. Climate, 24, 563-568, https://doi.org/10.1175/ 2010JCLI4077.1.

Lindzen, R. S., and A. V. Hou, 1988: Hadley circulations for zonally averaged heating centered off the equator. J. Atmos. Sci., 45, 2416-2427, https://doi.org/10.1175/1520-0469(1988)045<2416: $\mathrm{HCFZAH}>2.0 . \mathrm{CO} ; 2$.

Nguyen, H., A. Evans, C. Lucas, I. Smith, and B. Timbal, 2013: The Hadley circulation in reanalyses: Climatology, variability, and change. J. Climate, 26, 3357-3376, https://doi.org/10.1175/ JCLI-D-12-00224.1.

Randel, W. J., and I. M. Held, 1991: Phase speed spectra of transient eddy fluxes and critical layer absorption. J. Atmos. Sci., 48, 688-697, https://doi.org/10.1175/1520-0469(1991)048<0688: PSSOTE $>2.0 . \mathrm{CO} ; 2$.

Rodwell, M. J., and B. J. Hoskins, 2001: Subtropical anticyclones and summer monsoons. J. Climate, 14, 3192-3211, https:// doi.org/10.1175/1520-0442(2001)014<3192:SAASM>2.0.CO;2.

Schneider, T., 2006: The general circulation of the atmosphere. Annu. Rev. Earth Planet. Sci., 34, 655-688, https://doi.org/ 10.1146/annurev.earth.34.031405.125144.

— , and S. Bordoni, 2008: Eddy-mediated regime transitions in the seasonal cycle of a Hadley circulation and implications for monsoon dynamics. J. Atmos. Sci., 65, 915-934, https://doi.org/ 10.1175/2007JAS2415.1.

Seager, R., N. Harnik, W. A. Robinson, Y. Kushnir, M. Ting, H.-P. Huang, and J. Velez, 2005: Mechanisms of ENSO-forcing of hemispherically symmetric precipitation variability. Quart. J. Roy. Meteor. Soc., 131, 1501-1527, https://doi.org/10.1256/ qj.04.96.

Shaw, T. A., 2014: On the role of planetary-scale waves in the abrupt seasonal transition of the Northern Hemisphere general circulation. J. Atmos. Sci., 71, 1724-1746, https://doi.org/ 10.1175/JAS-D-13-0137.1

Stachnik, J. P., and C. Schumacher, 2011: A comparison of the Hadley circulation in modern reanalyses. J. Geophys. Res., 116, D22101, https://doi.org/10.1029/2011JD016677.

Vallis, G. K., P. Zurita-Gotor, C. Cairns, and J. Kidston, 2015: Response of the large-scale structure of the atmosphere to global warming. Quart. J. Roy. Meteor. Soc., 141, 1479-1501, https://doi.org/10.1002/qj.2456.

Walker, C. C., and T. Schneider, 2006: Eddy influences on Hadley circulations: Simulations with an idealized GCM. J. Atmos. Sci., 63, 3333-3350, https://doi.org/10.1175/JAS3821.1. 